\title{
Infográficos para decisão compartilhada no uso de estatinas em paciente de alto risco cardiovascular
}

\author{
Infographic for shared-decision for statins use in patients with high cardiovascular risk
}

\section{Infográficos para decisión compartida en pacientes con alto riesgo cardiovascular}

\author{
Fernanda Karolinne Melchior Silva Pinto', Jardel Corrêa de Oliveira² \\ ${ }_{1}^{1}$ Programa de Residência em MFC da Unievangelica/GO. Anápolis, GO, Brasil. \\ 2 Programa de Residencia em Medicina de Família e Comunidade da ESP; Secretaria Municipal de Saúde de Florianópolis. Florianópolis, SC, Brasil.
}

\section{Resumo}

Objetivos: Elaborar infográficos para decisão compartilhada sobre o benefício e risco do uso de estatinas como prevenção primária em pacientes com alto risco cardiovascular. Métodos: A partir do "the NNT", que analisou o uso de estatinas para prevenção primária em pessoas de baixo risco cardiovascular a partir de meta-análises do US Preventive Services Task Force (USPSTF) e da Cochrane, buscou-se definir o balanço de risco e benefício dessa terapia na prevenção primária de pessoas com alto risco. Como o USPSTF e a Cochrane tinham 10 estudos primários diferentes incluídos em suas análises e populações com risco cardiovascular variável, foi realizada uma busca adicional por revisões sistemáticas e meta-análises publicadas desde a data da revisão Cochrane (dezembro de 2012) até outubro de 2017. Buscou-se no Medline via PubMed, na Biblioteca Virtual em Saúde, na Cochrane e no NHS Evidence por estudos de pessoas com risco cardiovascular estimado em 10 anos de mais de $10 \%$ com mais um fator de risco ou com mais de $20 \%$, sem história de doença cardiovascular prévia, que comparassem o uso de estatina com placebo ou nenhum tratamento. Os desfechos deveriam ser a redução de doenças cardio e cerebrovasculares, da mortalidade cardiovascular ou geral e a ocorrência de eventos adversos. Resultados: Foram encontrados 225 estudos entre meta-análises e revisões sistemáticas elaboradas desde dezembro de 2012 até outubro de 2017. Foram excluídos 224 estudos por motivos diversos. Selecionou-se para elaboração do infográfico de benefícios o Statins for primary prevention, 2012, com população de $100 \%$ de diabéticos sem eventos cardiovasculares prévios com redução de risco absoluto (RRA) quanto a AVC fatal de 0,78 pontos percentuais/NNT 128 e Doenças Cardio/Cerebrovasculares RRA 2,6 pontos percentuais/NNT 39, cardiovascular. Quanto à redução de mortalidade geral (RR 0,79; IC95\% 0,58-1,08) e revascularização (RR 0,74; IC 95\% 0,55-1,00) não foi encontrada diferença estatística significante. Devido às limitações encontradas nas revisões sistemáticas avaliadas, não foi possível compilar dados, no formato de infográfico, sobre a incidência de diabetes e abandono de terapia. Assim, para elaboração da parte referente a danos, foi selecionado o ECR Aspen, com população de diabéticos de alto risco para desenvolvimento de eventos cardiovasculares, sem eventos prévios, quanto à mialgia com aumento do risco absoluto (ARA) de 1,43 pontos percentuais/NND 70 (RR 1,91; IC95\% 1,03-3,53). Conclusão: Foi elaborado infográfico, com e sem uso de estatina, para desfechos cardiocerebrovasculares não fatais, AVC fatal, necessidade de revascularização, mortalidade geral e para ocorrência adversa de sintomas musculares. Esse instrumento pode ser utilizado na Atenção Primária durante o encontro clínico visando à tomada de decisão compartilhada, facilitando a compreensão dos riscos e benefícios do uso de estatina em pacientes de alto risco cardiovascular (RCV $10 \%$ com pelo menos um fator de risco adicional ou $>20 \%$ em 10 anos), diabéticos, nos quais a prescrição de estatina seja cogitada.

Palavras-chave: Estatinas; Doenças Cardiovasculares; Transtornos Cerebrovasculares; Atenção à Primária à Saúde; Tomada de Decisões

Como citar: Pinto FKMS, Oliveira JC. Infográficos para decisão compartilhada no uso de estatinas em paciente de alto risco cardiovascular. Rev Bras Med Fam Comunidade. 2019;14(41):1809. https://doi.org/10.5712/rbmfc14(41)1809
Autor correspondente:

Fernanda Karolinne Melchior Silva Pinto. E-mail: fernanda_melchior@hotmail.com Fonte de financiamento: declaram não haver.

Parecer CEP: não se aplica.

Procedência e revisão por pares: revisado por pares. Recebido em: 05/07/2018. Aprovado em: 23/10/2018. 


\begin{abstract}
Objectives: To elaborate infographics for shared-decision on the benefit and risk of the use of statins as primary prevention in patients with high cardiovascular risk. Methods: From the "NNT", which analyzed the use of statins for primary prevention in low-risk cardiovascular individuals from meta-analyzes of the US Preventive Services Task Force (USPSTF) and Cochrane, we sought to define the risk balance and the benefit of such therapy in the primary prevention of people at high risk. Because the USPSTF and Cochrane had 10 different primary studies included in their analyzes and populations at varying cardiovascular risk, an additional search was conducted for systematic reviews and meta-analyzes published since the date of the Cochrane review (December 2012) through October 2017. We searched Medline via PubMed, the Virtual Health Library, Cochrane, and NHS Evidence for studies of people with a 10-year cardiovascular risk estimated at more than $10 \%$ with one more risk factor or more than $20 \%$ without history of previous cardiovascular disease, who compared statin use with placebo or no treatment. The outcomes should be the reduction of cardiovascular and cerebrovascular diseases, cardiovascular or general mortality and the occurrence of adverse events. Results: One hundred and twenty-five studies were found between meta-analyzes and site-based reviews from December 2012 to October 2017 . One hundred and twenty-four studies were excluded for several reasons. Statins for primary prevention, 2012, with a population of $100 \%$ of diabetics with no prior cardiovascular events with absolute risk reduction (RRA) for fatal stroke of 0.78 percentage points/NNT 128 and Cardio/Cerebrovascular Diseases RRA 2.6 percentage points/NNT 39, cardiovascular. Regarding the reduction of general mortality (RR $0.79,95 \% \mathrm{Cl} 0.58-1.08)$ and revascularization (RR $0.74,95 \% \mathrm{Cl} 0.55-1.00$ ), no significant statistical difference was found. Due to the limitations found in the systematic reviews evaluated, it was not possible to compile infographic data on the incidence of diabetes and abandonment of therapy. Thus, to elaborate the part related to damages, the Aspen ECR was selected, with a population of diabetics of high risk for the development of cardiovascular events, without previous events, as for myalgia with an increase in absolute risk (ARA) of 1.43 percentage points/NND 70 (RR 1.91, IC 95\% 1.03-3.53). Conclusion: An infographic was elaborated, with and without statin, for non-fatal cardiocerebrovascular outcomes, fatal stroke, need for revascularization, general mortality and for the adverse occurrence of muscular symptoms. This instrument can be used in Primary Care during the clinical meeting for shared decision making, facilitating the understanding of the risks and benefits of statin use in patients at high cardiovascular risk (RCV $10 \%$ with at least one additional risk factor or $>20 \%$ in 10 years), in whom the statin prescription is considered.
\end{abstract}

Keywords: Statins; Cardiovascular Diseases; Cerebrovascular Diseases; Primary Health Care; Decision Making

\title{
Resumen
}

Objetivos: Elaborar infográficos para una decisión compartida sobre el beneficio y riesgo del uso de estatinas como prevención primaria en pacientes con alto riesgo cardiovascular. Métodos: A partir del "NNT", que analizó el uso de estatinas para prevención primaria en personas de bajo riesgo cardiovascular a partir de meta-análisis del US Preventive Services Task Force (USPSTF) y de Cochrane, se buscó definir el balance de riesgo y beneficio de esta terapia en la prevención primaria de personas con alto riesgo. Como USPSTF y Cochrane tenían 10 estudios primarios diferentes incluidos en sus análisis y poblaciones con riesgo cardiovascular variable, se realizó una búsqueda adicional por revisiones sistemáticas y meta-análisis publicadas desde la fecha de la revisión Cochrane (diciembre de 2012) hasta octubre de 2017. Se buscó en Medline vía PubMed, en la Biblioteca Virtual en Salud, en Cochrane y en NHS Evidence por estudios de personas con riesgo cardiovascular estimado en 10 años de más del $10 \%$ con más un factor de riesgo o con más del $20 \%$, sin antecedentes de enfermedad cardiovascular previa, que comparasen el uso de estatina con placebo o ningún tratamiento. Los resultados deberían ser la reducción de enfermedades cardio y cerebrovasculares, de la mortalidad cardiovascular o general y la ocurrencia de eventos adversos. Resultados: Se encontraron ciento y veinticinco estudios entre los metanálisis y las revisiones basadas en el sitio desde diciembre de 2012 hasta octubre de 2017 . Se excluyeron ciento y veinticuatro estudios por varias razones. Estatinas para la prevención primaria, 2012, con una población del 100\% de diabéticos sin eventos cardiovasculares previos con reducción del riesgo absoluto (RRA) para accidente cerebrovascular mortal de 0,78 puntos porcentuales/NNT 128 y enfermedades cardiovasculares/cerebrovasculares RRA 2,6 puntos porcentuales/NNT 39, cardiovascular. En cuanto a la reducción de la mortalidad general (RR 0,79; IC del 95\%: 0,58 a 1,08) y la revascularización (RR 0,74; IC del 95\%: 0,55 a 1,00), no se encontraron diferencias estadísticas significativas. Debido a las limitaciones encontradas en las revisiones sistemáticas evaluadas, no fue posible compilar datos infográficos sobre la incidencia de diabetes y el abandono de la terapia. Así, para elaborar la parte relacionada con daños, se seleccionó el ECR de Aspen, con una población de diabéticos de alto riesgo para el desarrollo de eventos cardiovasculares, sin eventos previos, en cuanto a mialgia con un aumento del riesgo absoluto (ARA) del 1.43 por ciento. puntos/NND 70 (RR 1.91, IC 95\% 1.03-3.53). Conclusión: Se elaboró un infográfico, con y sin estatina, para resultados cardiocerebrovasculares no fatales, accidente cerebrovascular fatal, necesidad de revascularización, mortalidad general y para la aparición adversa de síntomas musculares. Este instrumento se puede utilizar en Atención Primaria durante la reunión clínica para la toma de decisiones compartida. Facilitar la comprensión de los riesgos y beneficios del uso de estatinas en pacientes con alto riesgo cardiovascular (RCV 10\% con al menos un factor de riesgo adicional o> $20 \%$ en 10 años), en quienes se considera la prescripción de estatinas.

Palabras clave: Estatinas; Enfermedades Cardiovasculares; Trastornos Cerebrovasculares; Atención Primaria de Salud; Toma de Decisiones 


\section{Introdução}

O uso da chamada decisão compartilhada - shared decision - vem sendo demonstrado como uma forma de melhorar as relações "médico-pacientes" nas consultas clínicas e facilitar a aderência aos tratamentos propostos, além de facilitar escolhas sobre realização de discutíveis prevenções e rastreios. ${ }^{1}$ Apesar de não existir um modelo único para sua aplicação, alguns preconizam que além do uso das habilidades de comunicação, a utilização de ferramentas de auxílio pode ser extremamente útil. Em especial, no que concerne a informar o paciente sobre as opções possíveis. ${ }^{1-3}$

Uma das ferramentas utilizadas neste contexto são os chamados infográficos: gráficos visuais que descrevem informações chaves de maneira lógica e simplificada utilizando elementos visuais, dados numéricos, textuais e figuras. ${ }^{4,5}$ Pretende-se, por meio destes, sintetizar informação científica, bem como dados estatísticos, para o entendimento do público. ${ }^{5}$

Atualmente, uma das questões clínicas em que o uso da decisão compartilhada se faz necessário, e para a qual uma ferramenta como infográfico pode ser utilizada, é a avaliação de riscos e benefícios sobre o uso de medicamentos para a prevenção primária e/ou secundária com seus efeitos adversos potenciais. ${ }^{4}$ Exemplo é o uso de estatinas e a correlação das mesmas com a prevenção de desfechos cardiovasculares, tema que vem gerando divergências em diversos diretrizes clínicas e revisões sistemáticas. ${ }^{4,6}$ Contudo, o conhecimento científico e a análise estatística muitas vezes se tornam de difícil compreensão, inclusive para o profissional de saúde, ainda mais quando esses dados devem ser utilizados para auxiliar o paciente em uma decisão compartilhada.

O "the NNT"- NNT (Number need to treat/Número necessário para tratar), ${ }^{7}$ buscando auxiliar em decisões clínicas como essas, fez uma avaliação de risco x benefícios do uso de estatina em pessoas de baixo risco cardiovascular, utilizando como base as revisões da US Preventive Services Task Force ${ }^{8}$ e Cochrane. ${ }^{9}$ Como ambas as revisões utilizam estudos com pessoas de risco cardiovascular variável e na ausência de informações semelhantes às do "the NNT" para pessoas de alto risco cardiovascular (RCV), percebeu-se a necessidade de buscar e sumarizar dados referentes ao risco e o benefício do uso de estatina na prevenção primária neste grupo.

Assim, o objetivo deste artigo é elaborar infográficos para decisão compartilhada sobre o uso de estatinas como prevenção primária em pacientes com alto risco cardiovascular.

\section{Métodos}

Os autores iniciaram a avaliação a partir da atualização recente do tópico realizada pela US Preventive Services Task Force, ${ }^{8}$ 2016, e pelo "the NNT", ${ }^{7} 2017$, comparando este estudo com a revisão elaborada pela Cochrane. As avaliações do "the NNT" e do USPSTF são mais recentes, mas apresentaram 10 estudos diferentes considerados entre a revisão Cochrane e do USPSTF (Tabela 1). Dessa maneira, optou-se por buscar outras meta-análises e revisões sistemáticas publicadas após a da Cochrane para avaliar a inclusão de algum outro estudo de interesse. 
Tabela 1. Estudos primários incluídos nas meta-análises avaliadas com as respectivas amostras avaliadas.

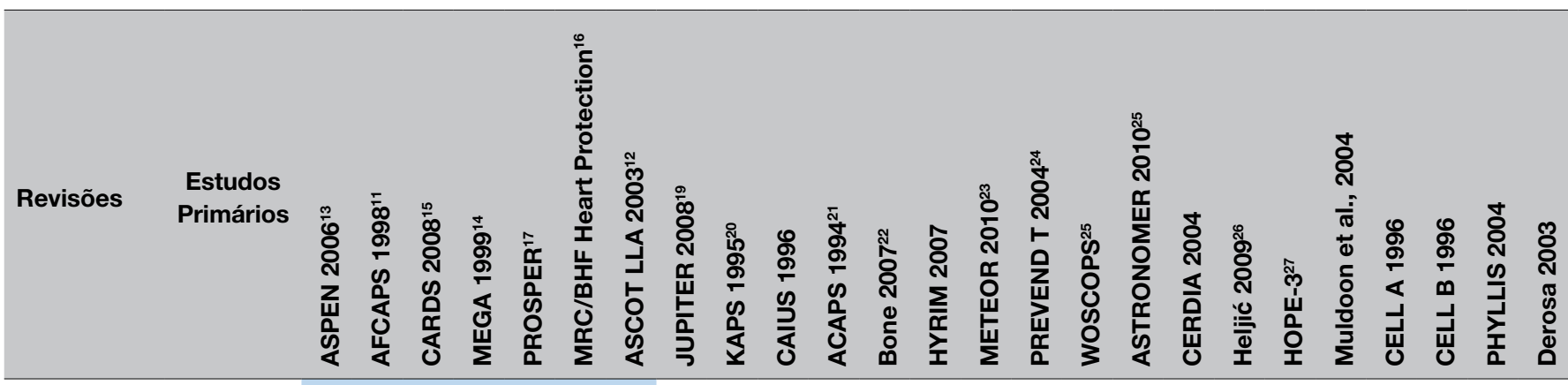

\begin{tabular}{|c|c|c|c|c|c|}
\hline $\begin{array}{l}\text { Statins for } \\
\text { Primary } \\
\text { prevention }^{10 *}\end{array}$ & $\begin{array}{l}7 \text { ECR's } \\
\text { Estatinas/ } \\
\text { Controle }\end{array}$ & 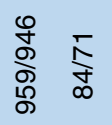 & 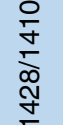 & $\begin{array}{l}\stackrel{\mathscr{D}}{\infty} \\
\stackrel{\infty}{\infty} \\
\stackrel{0}{\infty} \\
\infty\end{array}$ & 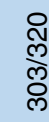 \\
\hline
\end{tabular}

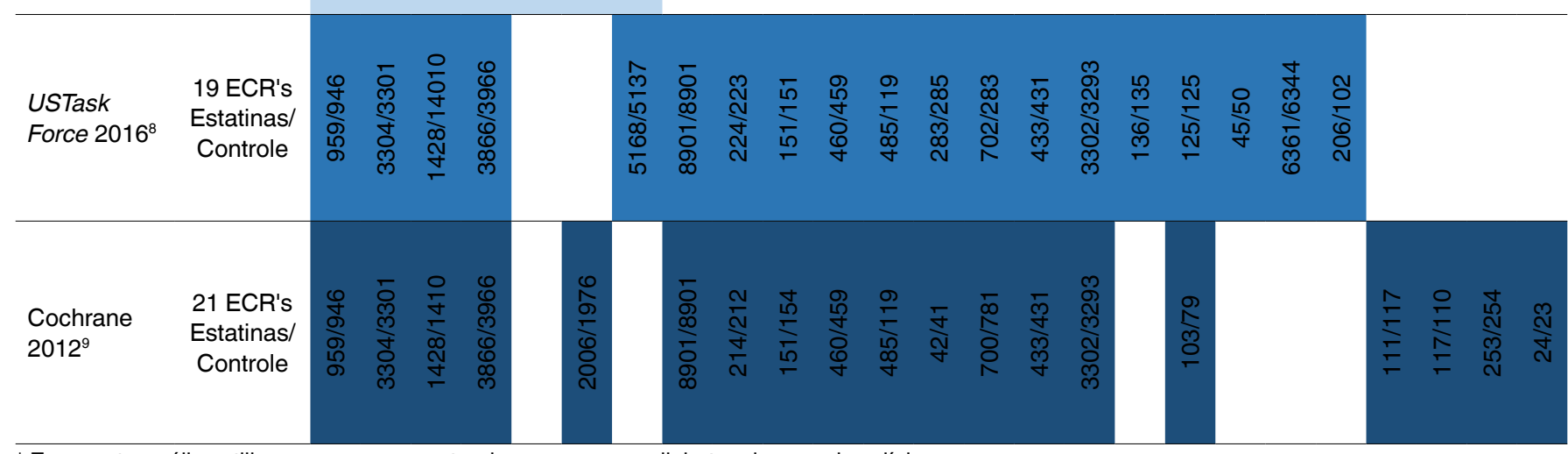

* Essa meta-análise utilizou apenas a amostra de pessoas com diabetes dos ensaios clínicos.

A pergunta de pesquisa, de acordo com o modelo PICO, foi:

$\mathrm{P}$ - Pessoas com alto risco de eventos cardiovasculares sem doença cardiovascular prévia

I - Estatina

C - Placebo ou nenhum medicamento

O - Eventos Cardiovasculares como ocorrência de infarto agudo do miocárdio, acidente vascular cerebral, doença arterial obstrutiva periférica, mortalidade cardiovascular ou geral e ocorrência de efeitos adversos.

Definiu-se como alto risco aqueles com risco estimado em 10 anos maior que $10 \%$ com mais um fator de risco, conforme adotado pelo USPSTF, ou maior que $20 \%$.

Realizou-se busca por revisões sistemáticas e meta-análises no PubMed, Biblioteca Virtual em Saúde (exceto Medline), Cochrane, NHS Evidence, por estudos publicados de dezembro de 2012 (data da revisão Cochrane) até outubro de 2017. Não houve restrição de idioma, sendo os limites: humanos e tipo de estudo. A qualidade dos estudos selecionados foi avaliada segundo as diretrizes metodológicas para elaboração de pareceres técnico-científicos do Ministério da Saúde.

A estratégia de busca com descritores em saúde do Mesh e sinônimos: (hydroxymethylglutaryl coa reductase inhibitors) AND ("Myocardial infarction" OR "Angina, unstable" OR "Coronary disease" OR "Coronary artery disease" OR "acute coronary syndrome" OR "Coronary stenosis" OR "Coronary restenosis" OR "Coronary occlusion" OR "Coronary thrombosis" OR "angioplasty" OR "mortality" OR "death, sudden, 
cardiac" OR stroke OR "Cerebral Stroke" OR "Cerebrovascular Accident" OR "Acute Cerebrovascular Accident" OR "Cerebrovascular Stroke" OR "Acute Stroke" OR "Brain Vascular Accident" OR "Peripheral Arterial Disease").

\section{Resultados e Discussão}

Foram encontrados 225 estudos entre meta-análises e revisões sistemáticas elaboradas desde dezembro de 2012 até outubro de 2017. Desses, 12 foram excluídos por ser outro tipo de estudo. Outras 105 foram eliminadas pela leitura do título e resumo, dos quais 58 por apresentarem população e avaliação de situações clínicas variadas (doença pulmonar obstrutiva crônica, pneumonia, transplante cardíaco e sepse) e 47 por avaliar intervenções múltiplas (estatina + revascularização ou dieta ou anti-hipertensivo, etc.). Outros 89 por envolverem desfechos distintos dos selecionados pela pergunta de pesquisa ou correlacionados com avaliação de parâmetros genéticos e/ou baseados em exames complementares. Por último, foram excluídos quatro duplicados e cinco que continham estudos primários com população total já incluída nas demais revisões sistemáticas (Fluxograma 1).

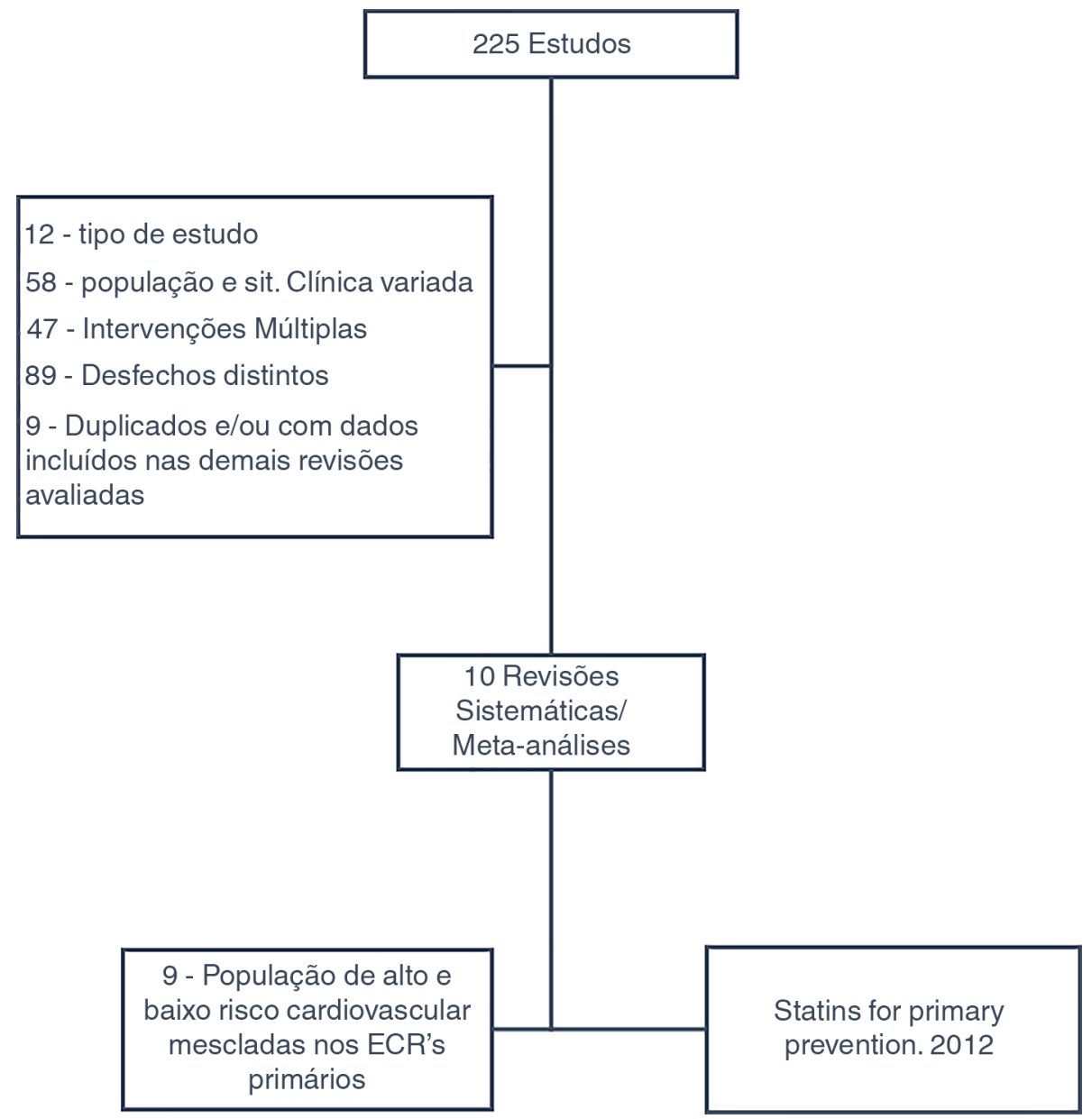

Fluxograma 1. Motivos de inclusão e exclusão das revisões sistemáticas e meta-análises conforme critérios do estudo. 
Grande parte das revisões sistemáticas e meta-análises baseou-se em estudos primários com tipos variados de população (comorbidades, sexo, faixas etárias, níveis variáveis de alteração do lipidograma, incluindo até $10 \%$ de pessoas com ocorrência de eventos cardiovasculares prévios) submetidas a diferentes tipos de intervenções (sinvastatina, atorvastatina, pravastatina, lovastatina, rosuvastatina), avaliando diferentes tipos de desfecho. Assim, nove revisões foram descartadas, pois envolviam pessoas de baixo e alto risco cardiovascular, não sendo possível extrair dados especificamente da população de alto risco para a elaboração dos infográficos.

Ao final, foi selecionada uma meta-análise (Statins for primary prevention), ${ }^{10}$ que avaliou 7 ensaios clínicos controlados e randomizados (ECR), ${ }^{11-17}$ com um total de 12.711 pacientes diabéticos, dos quais 6340 estavam alocadas no grupo intervenção. Apesar de representar uma população de alto risco cardiovascular, isso pode representar uma limitação quanto à validade externa dos resultados, uma vez que possivelmente não se aplicam a pessoas com alto risco que não tenham diabetes. As revisões avaliadas de maneira geral ${ }^{8-10}$ apresentam vários estudos em comum, com populações de variados riscos cardiovasculares (Tabela 1).

Para elaboração de infográficos que auxiliem na individualização do processo de tomada de decisão compartilhada durante o encontro clínico, tanto os possíveis benefícios quanto os riscos devem ser discutidos.

\section{Benefícios}

A meta-análise selecionada ${ }^{10}$ para elaboração do infográfico (Infográfico 1) apresentou um risco absoluto (RA) de 2,78\% com placebo e $2 \%$ com estatina para ocorrência de acidente vascular cerebral (AVC) fatal, com uma redução de risco absoluto (RRA) de 0,78 pontos percentuais (RR 0,71; IC 95\% 0,54 - 0,94; NNT 128). Para doenças cardiocerebrovasculares não fatais, houve um RA de $12,1 \%$ com placebo e 9,54\% com estatina, com RRA de 2,6 pontos percentuais (RR 0,79; IC95\% 0,66-0,95; NNT 39). As doenças cardiocerebrovasculares incluíam infarto agudo do miocárdio (IAM) não fatal, AVC não fatal e acidente isquêmico transitório. Não foi encontrada significância estatística na redução de mortalidade geral ( $R R$ 0,79 IC95\% 0,58-1,08) e quanto à necessidade de revascularização ( $R R$ 0,74; IC 95\% 0,55-1,00), com riscos absolutos respectivamente de 4,2\% e 2,6\%. É importante considerar que o benefício citado para as doenças cardiocerebrovasculares é idêntico ao NNT de 39 descrito pelo "the NNT" para prevenção secundária de IAM não fatal. ${ }^{18}$ Como tais doenças representam um desfecho composto, o NNT de 39 nesse contexto pode ter sido superestimado.

\section{Riscos}

Para determinar os riscos não foi encontrada nenhuma revisão sistemática ou meta-análise, com população de alto risco cardiovascular, na qual a prescrição de estatina fosse indicada como prevenção primária em sua totalidade. Alguns grandes estudos ${ }^{28,29}$ que encontraram como efeitos adversos miopatia, elevação de transaminases, aumento na incidência de diabetes e câncer apresentam população com risco cardiovascular estimado altamente variável (RCV $<5 \%$ a $>40 \%$ ), o que dificulta a quantificação desses riscos na população delimitada para construção dos infográficos nesse estudo. Dessa maneira, optou-se por avaliar as evidências disponíveis nos ECR encontrados nas revisões sistemáticas analisadas, para diferentes eventos adversos. 
K

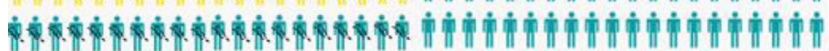

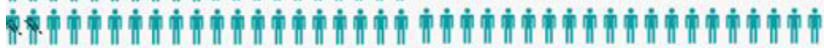

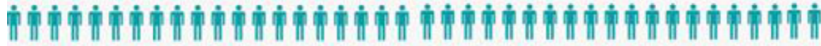

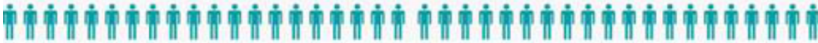

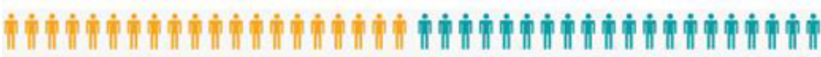

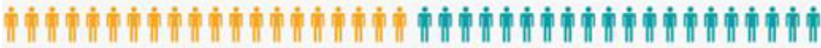

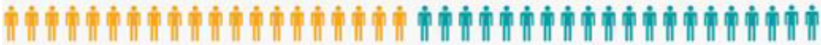

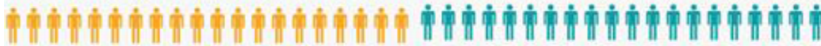

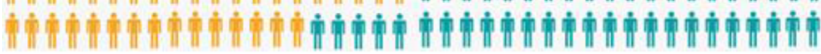

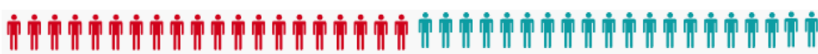

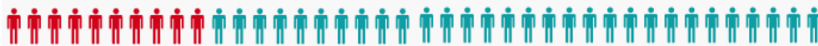

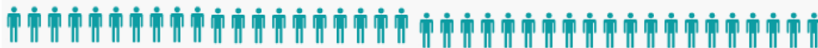

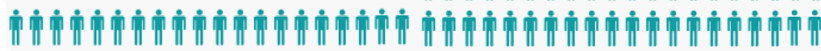

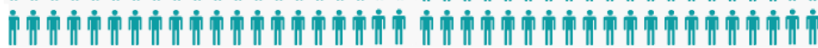

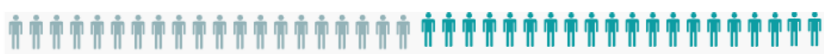

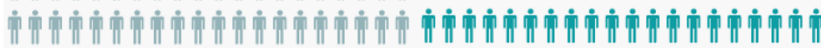

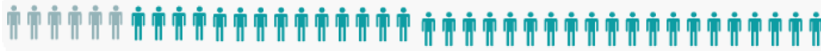

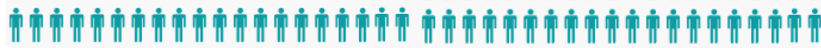

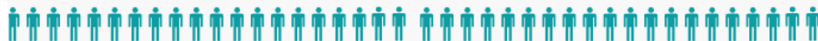

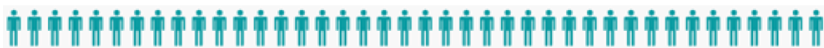

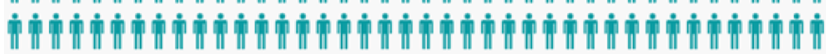

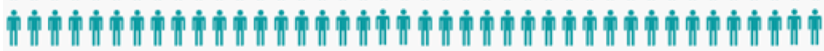

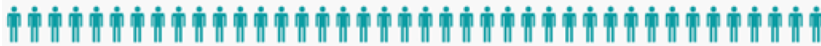

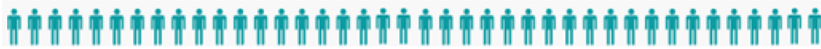

in

I 1000 Pessoas com alto RCV em uso de Estatina por 3 a 5 anos

95 pessoas com DCCV não fatal

20 mortes por AVC

"i 30 pessoas com sintomas musculares adversos

"| 26 pessoas submetidas a revascularização

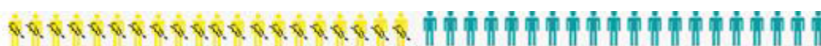

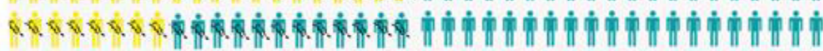

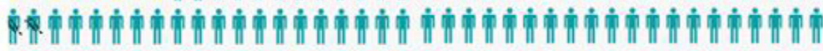

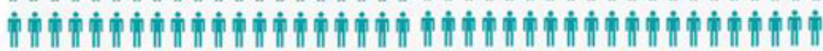

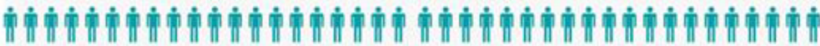

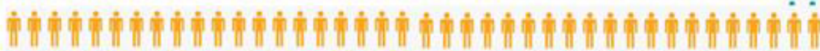

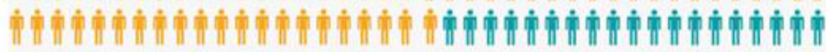

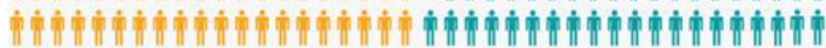

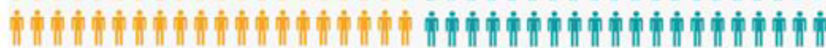

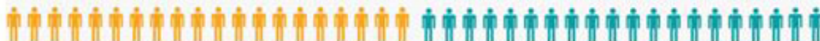

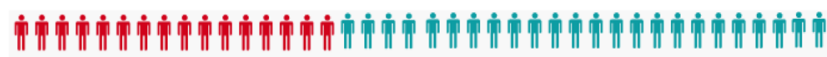

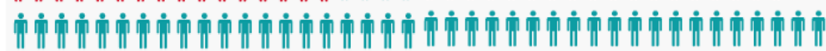

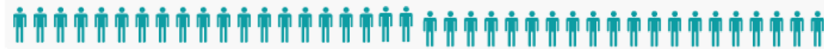

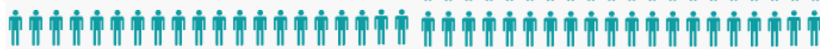

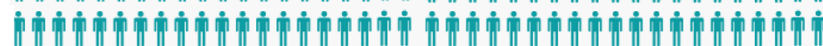

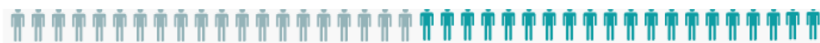

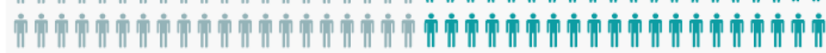

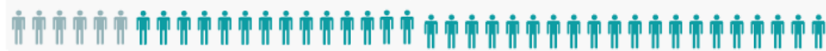

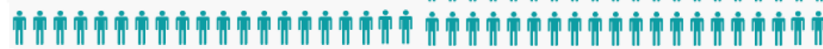

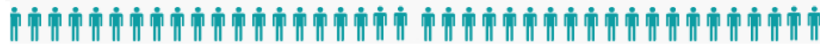

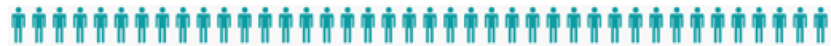

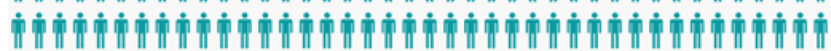

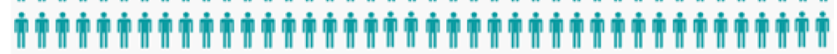

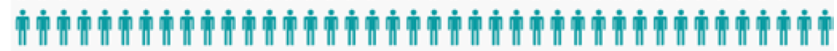

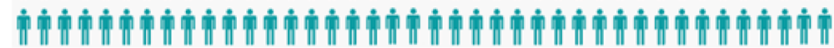

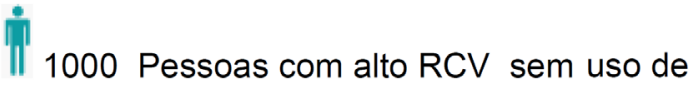
Estatina por 3 a 5 anos

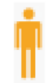

121 pessoas com DCCV não fatal

28 mortes por AVC

II 16 pessoas com sintomas musculares adversos

Q 42 mortes por causas gerais

Infográfico 1. Infográfico para decisão compartilhada sobre uso de estatinas para prevenção primária de pessoas com diabetes e alto risco cardiovascular.

RCV: Risco Cardiovascular; DCCV: Doenças Cerebrocardiovasculares (IAM e AVC não fatal e AIT); AVC: Acidente Vascular Cerebral. 


\section{Incidência de Diabetes Mellitus (DM)}

O ECR Jupiter ${ }^{30}$ é conhecido como um dos maiores estudos, inclusive sendo a base para os dados do " the NNT"7 e incluído pela USPSTF" e pela Cochrane. ${ }^{9}$ Isoladamente, apontou a correlação entre uso de estatinas e o aumento da incidência de DM tipo 2 como desfecho decorrente do uso de Rosuvastatina em alta dose (RR 1,18 IC95 1,01-1,39), com um NND 204 -NND (número necessário para causar dano). ${ }^{19}$ No entanto, este ECR avaliou população de risco variável (RCV de 7,3\%, em mulheres não tabagistas, até $23,7 \%$, em homens tabagistas), o que não permitiu seu uso para elaboração do infográfico.

A revisão sistemática mais recente, US Preventive Services Task Force,${ }^{8}$ conta com 19 ECR, avaliando um total de 71.344 pacientes maiores de 40 anos, em sua maioria sem doença cardiovascular prévia. A revisão optou por selecionar estudos que incluíam no máximo 10\% de pessoas com DCV prévia. Os ECR foram divididos em diferentes grupos para análise de variados riscos e em nenhum deles foi encontrada diferença significativa estatisticamente. Para o cálculo de incidência de DM especificamente, foram utilizados seis ECR, ${ }^{11,12,14,19,27,31}$ com um total de 59.083 pacientes (RR 1,05; IC95\% 0,91-1,2). No entanto, desses seis ECR, um tinha $18 \%$ de pessoas com doença cardiovascular prévia, ${ }^{12}$ o que contraria o critério de seleção do próprio USPSTF, e motivou sua exclusão da revisão Cochrane. ${ }^{9}$ Outro ${ }^{14}$ não apresentou dados sobre a incidência de DM, não tendo sido inclusive utilizado pela Cochrane para avaliar esse desfecho em sua revisão. Assim, não foi possível determinar como os autores do UPSTF obtiveram esses dados.

A revisão Cochrane analisou conjuntamente os ECR Jupiter e AFCAPS, ${ }^{11}$ encontrando uma RRA de 0,43 pontos percentuais (RR 1,18 IC 95\% 1,01-1,39; NND 235). No entanto, apesar de incluir o estudo WOSCOPS ${ }^{31}$ em outras análises, não considerou seus dados sobre a redução da incidência de DM com uso de pravastatina, os quais foram avaliados na meta-análise da USPSTF. ${ }^{8}$ Outra limitação para que se pudesse considerar os dados combinados da Cochrane ${ }^{9}$ é que o período até o qual se estendeu a busca por seus artigos primários não incluiu o ECR HOPE-3 ${ }^{32}$ publicado posteriormente e incluído pelo USPSTF.

Assim, ambas as meta-análises, além de envolverem pessoas com RCV variável, possuem limitações em suas meta-análises sobre a incidência de DM. Portanto, não foram consideradas adequadas para a elaboração do infográfico.

\section{Sintomas musculares}

A revisão Cochrane não encontrou diferença estatisticamente significativa quanto à ocorrência de miopatia e rabdomiólise entre estatina e placebo (RR 1,03; IC 95\% 0,97-1,09). ${ }^{9}$ O US Preventive Services Task Force também não mostrou diferença para mialgia (RR 0,96; IC95\% 0,79-1,16).

Alguns estudos, patrocinados pela indústria farmacêutica, discutem que a alta ocorrência de efeitos adversos como miopatia, relatada em estudos observacionais, levando em alguns casos a suspensão da estatina, estejam associadas ao chamado efeito nocebo (aumento da ocorrência de efeitos adversos quando paciente e médico acreditavam que paciente estava em uso do medicamento). ${ }^{33,34}$

O ECR Aspen, ${ }^{13}$ incluído nas três meta-análises, ${ }^{8-10}$ avaliou 2410 pessoas com DM e alto risco cardiovascular, sem eventos cardiovasculares prévios, que usaram atorvastatina $10 \mathrm{mg}$ como prevenção primária e encontrou RA de $1,59 \%$ no grupo placebo e de $3,02 \%$ no de estatina, com aumento de risco absoluto de 1,43 pontos percentuais (RR 1,91; IC95\% 1,03-3,53; NND 70). Contudo, no grupo placebo do 
estudo, cerca de $30 \%$ fazia uso de outros redutores de lipídios, o que pode ter aumentado a ocorrência de efeitos adversos no grupo "placebo", podendo subestimar a RRA e, consequentemente, aumentar o NND. De qualquer forma, por ser o único ECR que avaliou esse efeito adverso na população alvo deste estudo, optou-se por utilizá-lo na construção do infográfico (Infográfico 1).

\section{Abandono de terapia}

Evidências anteriores apontavam a ocorrência de efeitos adversos como uma das causas para abandono da terapia com estatinas. ${ }^{35}$ Meta-análise recente, que avaliou 130.000 pacientes entre grupo em uso de estatina (média e alta intensidade) e placebo, evidenciou taxas semelhantes de abandono em ambos os grupos sem relevância estatística (OR 0,99; IC 95\% 0,93-1,06). ${ }^{12}$

Conclui-se que as evidências acerca dos efeitos adversos sobre o uso de estatina são conflituosas em grande parte das meta-análises e revisões sistemáticas, algumas com problemas com viés de seleção, outras com viés de alocação, outras com variáveis de confusão, o que impossibilitou o uso de suas informações no infográfico. Assim, os dados sobre efeitos adversos do infográfico foram baseados em um ECR com população diabética de alto risco cardiovascular sem evento prévio. ${ }^{10}$

\section{Conclusão}

Uma decisão compartilhada coerente deve ser realizada quando existe recomendação de utilizar a estatina (estimativa de RCV $>10 \%+1 \mathrm{FR}$ ou $>20 \%$ ), compartilhando de maneira clara os riscos e benefícios esperados em pacientes com risco cardiovascular semelhante ao da pessoa em questão.

Para apresentação dos benefícios e riscos, foi elaborado um infográfico. Com base neste, pode-se perceber que, apesar do pequeno benefício na redução de AVC fatal, não há diferença na mortalidade geral. É preciso lembrar que a diminuição vista na ocorrência de doenças cardiocerebrovasculares não fatais decorre de um desfecho composto, cujo resultado alcançado com uso e estatinas pode estar superestimado. Quanto à ocorrência de efeitos adversos, destaca-se a miopatia, não sendo possível utilizar dados sobre a incidência de novos casos de DM devido a limitações das meta-análises e ausência de ECR especificamente em população de alto risco cardiovascular que avaliassem esse efeito adverso.

É importante ressaltar a escassez ou mesmo ausência de estudos com uso de estatina na prevenção primária de pessoas com alto risco cardiovascular que não tenham diabetes.

Ainda assim, o uso do instrumento proposto pode ser de grande valia na atenção primária para tomada de decisão compartilhada com pessoas diabéticas com alto risco cardiovascular, inclusive quanto à melhora na aderência ao tratamento daqueles que optem por realizá-lo. ${ }^{36}$ Tal instrumento sintetiza a informação sobre o número de pessoas necessárias a serem tratadas para que uma pessoa seja beneficiada (NNT) com o número de pessoas que sofrem dano com o tratamento (NND), podendo facilitar a compreensão do paciente.

Ressalta-se que, apesar de facilitar e, inclusive, poder aumentar a adesão nos grupos especificados, infere-se que o instrumento pode apresentar limitações quanto à utilização em pacientes com déficits visuais. Tais dificuldades provavelmente serão encontradas para a realização de decisão compartilhada com ou sem o uso do instrumento. 
Ensaios clínicos sobre uso de estatinas exclusivamente para prevenção primária, estratificados de acordo com baixo ou alto risco cardiovascular, que envolvam não somente pessoas com diabetes no caso de estudos com pessoas de alto risco, e com qualidade metodológica, tempo de seguimento e avaliação de efeitos adversos adequados, são necessários para construção de ferramentas melhores que permitam a decisão compartilhada para diferentes grupos de pacientes nesse contexto.

\section{Conflito de interesses}

Declaram não haver.

\section{Contribuição dos autores}

Concepção e/ou delineamento do estudo: JF, FM. Aquisição, análise e interpretação dos dados: JF, FM. Redação preliminar: FM. Revisão crítica da versão preliminar: JF.

Todos os autores aprovaram a versão final e concordaram com prestar contas sobre todos os aspectos do trabalho.

\section{Referências}

1. Bouniols N, Leclère B, Moret L. Evaluating the quality of shared decision making during the patient-carer encounter: a systematic review of tools. BMC Res Notes. 2016;9:382. https://doi.org/10.1186/s13104-016-2164-6

2. Lawani MA, Valéra B, Fortier-Brochu É, Légaré F, Carmichael PH, Côté L, et al. Five shared decision-making tools in 5 months: use of rapid reviews to develop decision boxes for seniors living with dementia and their caregivers. Syst Rev. 2017;6(1):56. https://doi.org/10.1186/ s13643-017-0446-2

3. Légaré F, Hébert J, Goh L, Lewis KB, Leiva Portocarrero ME, Robitaille H, et al. Do choosing wisely tools meet criteria for patient decision aids? A descriptive analysis of patient materials. BMJ Open. 2016;6(8):e011918.

4. Sheridan SL, Draeger LB, Pignone MP, Rimer B, Bangdiwala SI, Cai J, et al. The effect of a decision aid intervention on decision making about coronary heart disease risk reduction: secondary analyses of a randomized trial. BMC Med Inform Decis Mak. 2014;14:14. https:// doi.org/10.1186/1472-6947-14-14

5. Scott H, Fawkner S, Oliver C, Murray A. Why healthcare professionals should know a little about infographics. Br J Sports Med [Internet]. 2016 Sep; [cited 2017 Jul 13]; 50(18):1104-5. Available from: http://bjsm. bmj.com/content/50/18/1104

6. Barrett B, Ricco J, Wallace M, Kiefer D, Rakel D. Communicating statin evidence to support shared decision-making. BMC Fam Pract. 2016;17:41. https://doi.org/10.1186/s12875-016-0436-9

7. Group GW and TN. Statins in Persons at Low Risk of Cardiovascular Disease [Internet]. The NNT. [cited 2018 Feb 6]. Available from: http://www.thennt.com/nnt/statins-persons-low-risk-cardiovascular-disease/

8. Chou R, Dana T, Blazina I, Daeges M, Jeanne TL. Statins for Prevention of Cardiovascular Disease in Adults: Evidence Report and Systematic Review for the US Preventive Services Task Force. JAMA. 2016;316(19):2008-24. https://doi.org/10.1001/jama.2015.15629

9. Taylor F, Huffman MD, Macedo AF, Moore TH, Burke M, Davey Smith G, et al. Statins for the primary prevention of cardiovascular disease. In: Cochrane Database of Systematic Reviews [Internet]. Hoboken: John Wiley \& Sons; 2013. Available from: http://onlinelibrary.wiley. com/doi/10.1002/14651858.CD004816.pub5/abstract

10. Chen YH, Feng B, Chen ZW. Statins for primary prevention of cardiovascular and cerebrovascular events in diabetic patients without established cardiovascular diseases: a meta-analysis. Exp Clin Endocrinol Diabetes. 2012;120(2):116-20. https://doi. org/10.1055/s-0031-1297968

11. Downs JR, Clearfield M, Weis S, Whitney E, Shapiro DR, Beere PA, et al. Primary prevention of acute coronary events with lovastatin in men and women with average cholesterol levels: results of AFCAPS/TexCAPS. Air Force/Texas Coronary Atherosclerosis Prevention Study. JAMA. 1998;279(20):1615-22. https://doi.org/10.1001/jama.279.20.1615 
12. Sever PS, Dahlöf B, Poulter NR, Wedel H, Beevers G, Caulfield M, et al. Prevention of coronary and stroke events with atorvastatin in hypertensive patients who have average or lower-than-average cholesterol concentrations, in the Anglo-Scandinavian Cardiac Outcomes Trial--Lipid Lowering Arm (ASCOT-LLA): a multicentre randomised controlled trial. Lancet. 2003;361(9364):1149-58. https://doi.org/10.1016/ S0140-6736(03)12948-0

13. Knopp RH, d'Emden M, Smilde JG, Pocock SJ. Efficacy and safety of atorvastatin in the prevention of cardiovascular end points in subjects with type 2 diabetes: the Atorvastatin Study for Prevention of Coronary Heart Disease Endpoints in non-insulin-dependent diabetes mellitus (ASPEN). Diabetes Care. 2006;29(7):1478-85. https://doi.org/10.2337/dc05-2415

14. Nakamura H, Arakawa K, Itakura H, Kitabatake A, Goto Y, Toyota T, et al.; MEGA Study Group. Primary prevention of cardiovascular disease with pravastatin in Japan (MEGA Study): a prospective randomised controlled trial. Lancet. 2006;368(9542):1155-63. https:// doi.org/10.1016/S0140-6736(06)69472-5

15. Colhoun HM, Betteridge DJ, Durrington PN, Hitman GA, Neil HA, Livingstone SJ, et al.; CARDS investigators. Primary prevention of cardiovascular disease with atorvastatin in type 2 diabetes in the Collaborative Atorvastatin Diabetes Study (CARDS): multicentre randomised placebo-controlled trial. Lancet. 2004;364(9435):685-96. https://doi.org/10.1016/S0140-6736(04)16895-5

16. Collins R, Armitage J, Parish S, Sleigh P, Peto R; Heart Protection Study Collaborative Group. MRC/BHF Heart Protection Study of cholesterol-lowering with simvastatin in 5963 people with diabetes: a randomised placebo-controlled trial. Lancet. 2003;361(9374):200516. https://doi.org/10.1016/S0140-6736(03)13636-7

17. Shepherd J, Blauw GJ, Murphy MB, Bollen EL, Buckley BM, Cobbe SM, et al.; PROSPER study group. PROspective Study of Pravastatin in the Elderly at Risk. Pravastatin in elderly individuals at risk of vascular disease (PROSPER): a randomised controlled trial. Lancet. 2002;360(9346):1623-30. https://doi.org/10.1016/S0140-6736(02)11600-X

18. Group GW and TN. Statins for 5 Heart Disease Prevention (With Known Heart Disease) [Internet]. The NNT. [cited 2018 Feb 7]. Available from: http://www.thennt.com/nnt/statins-for-heart-disease-prevention-with-known-heart-disease/

19. Ridker PM, Pradhan A, MacFadyen JG, Libby P, Glynn RJ. Cardiovascular benefits and diabetes risks of statin therapy in primary prevention: an analysis from the JUPITER trial. Lancet. 2012;380(9841):565-71. https://doi.org/10.1016/S0140-6736(12)61190-8

20.Salonen R, Nyyssönen K, Porkkala E, Rummukainen J, Belder R, Park JS, et al. Kuopio Atherosclerosis Prevention Study (KAPS). A population-based primary preventive trial of the effect of LDL lowering on atherosclerotic progression in carotid and femoral arteries. Circulation. 1995;92(7):1758-64. https://doi.org/10.1161/01.CIR.92.7.1758

21. Furberg CD, Adams HP Jr, Applegate WB, Byington RP, Espeland MA, Hartwell T, et al. Effect of lovastatin on early carotid atherosclerosis and cardiovascular events. Asymptomatic Carotid Artery Progression Study (ACAPS) Research Group. Circulation. 1994;90(4):1679-87. https://doi.org/10.1161/01.CIR.90.4.1679

22. Bone HG, Kiel DP, Lindsay RS, Lewiecki EM, Bolognese MA, Leary ET, et al. Effects of atorvastatin on bone in postmenopausal women with dyslipidemia: a double-blind, placebo-controlled, dose-ranging trial. J Clin Endocrinol Metab. 2007;92(12):4671-7. https://doi. org/10.1210/jc.2006-1909

23. Crouse JR 3rd, Raichlen JS, Riley WA, Evans GW, Palmer MK, O'Leary DH, et al.; METEOR Study Group. Effect of rosuvastatin on progression of carotid intima-media thickness in low-risk individuals with subclinical atherosclerosis: the METEOR Trial. JAMA. 2007;297(12):1344-53. https://doi.org/10.1001/jama.297.12.1344

24. Asselbergs FW, Diercks GF, Hillege HL, van Boven AJ, Janssen WM, Voors AA, et al.; Prevention of Renal and Vascular Endstage Disease Intervention Trial (PREVEND IT) Investigators. Effects of fosinopril and pravastatin on cardiovascular events in subjects with microalbuminuria. Circulation. 2004;110(18):2809-16. https://doi.org/10.1161/01.CIR.0000146378.65439.7A

25. Chan KL, Teo K, Dumesnil JG, Ni A, Tam J; ASTRONOMER Investigators. Effect of Lipid lowering with rosuvastatin on progression of aortic stenosis: results of the aortic stenosis progression observation: measuring effects of rosuvastatin (ASTRONOMER) trial. Circulation. 2010;121(2):306-14. https://doi.org/10.1161/CIRCULATIONAHA.109.900027

26. Heljić B, Velija-Asimi Z, Kulić M. The statins in prevention of coronary heart diseases in type 2 diabetics. Bosn J Basic Med Sci. 2009;9(1):716. https://doi.org/10.17305/bjbms.2009.2860

27. Effects of ramipril on cardiovascular and microvascular outcomes in people with diabetes mellitus: results of the HOPE study and MICROHOPE substudy. Heart Outcomes Prevention Evaluation Study Investigators. Lancet. 2000;355(9200):253-9.

28. Mills EJ, Wu P, Chong G, Ghement I, Singh S, AkI EA, et al. Efficacy and safety of statin treatment for cardiovascular disease: a network meta-analysis of 170,255 patients from 76 randomized trials. QJM. 2011;104(2):109-24. https://doi.org/10.1093/qjmed/hcq165 
29. Naci H, Brugts J, Ades T. Comparative tolerability and harms of individual statins: a study-level network meta-analysis of 246955 participants from 135 randomized, controlled trials. Circ Cardiovasc Qual Outcomes. 2013;6(4):390-9. https://doi.org/10.1161/ CIRCOUTCOMES.111.000071

30. Ridker PM, Danielson E, Fonseca FA, Genest J, Gotto AM Jr, Kastelein JJ, et al.; JUPITER Study Group. Rosuvastatin to prevent vascular events in men and women with elevated C-reactive protein. N Engl J Med. 2008;359(21):2195-207. https://doi.org/10.1056/ NEJMoa0807646

31. Shepherd J. The West of Scotland Coronary Prevention Study: a trial of cholesterol reduction in Scottish men. Am J Cardiol. 1995;76(9):113C-7.

32. Yusuf S, Bosch J, Dagenais G, Zhu J, Xavier D, Liu L, et al. Cholesterol Lowering in Intermediate-Risk Persons without Cardiovascular Disease. N Engl J Med. 2016;374(21):2021-31. https://doi.org/10.1056/NEJMoa1600176

33. Pedro-Botet J, Rubiés-Prat J. Statin-associated muscle symptoms: beware of the nocebo effect. Lancet. 2017;389(10088):2445-6. https:// doi.org/10.1016/S0140-6736(17)31163-7

34. Gupta A, Thompson D, Whitehouse A, Collier T, Dahlof B, Poulter N, et al.; ASCOT Investigators. Adverse events associated with unblinded, but not with blinded, statin therapy in the Anglo-Scandinavian Cardiac Outcomes Trial-Lipid-Lowering Arm (ASCOT-LLA): a randomised double-blind placebo-controlled trial and its non-randomised non-blind extension phase. Lancet. 2017;389(10088):2473-81. https://doi. org/10.1016/S0140-6736(17)31075-9

35. Zhang H, Plutzky J, Skentzos S, Morrison F, Mar P, Shubina M, et al. Discontinuation of statins in routine care settings: a cohort study. Ann Intern Med. 2013;158(7):526-34. https://doi.org/10.7326/0003-4819-158-7-201304020-00004

36. Weymiller AJ, Montori VM, Jones LA, Gafni A, Guyatt GH, Bryant SC, et al. Helping patients with type 2 diabetes mellitus make treatment decisions: statin choice randomized trial. Arch Intern Med [Internet]. 2007 May; [cited 2018 Feb 4]; 167(10):1076-82. Available from: https://www.ncbi.nlm.nih.gov/pubmed/17533211 